BULLETIN OF THE

AMERICAN MATHEMATICAL SOCIETY

Volume 79, Number 4, July 1973

\title{
SELFADJOINT SUBSPACE EXTENSIONS OF NONDENSELY DEFINED SYMMETRIC OPERATORS ${ }^{1}$
}

\author{
BY EARL A. CODDINGTON
}

Communicated by Fred Brauer, December 18, 1972

1. Subspaces in $\mathfrak{H}^{2}$. Let $\mathfrak{H}$ be a Hilbert space over the complex field $C$, and let $\mathfrak{H}^{2}=\mathfrak{H} \oplus \mathfrak{H}$ be the Hilbert space of all pairs $\{f, g\}$, where $f, g \in \mathfrak{H}$, with the inner product $(\{f, g\},\{h, k\})=(f, h)+(g, k)$. A subspace $T$ in $\mathfrak{H}^{2}$ is a closed linear manifold in $\mathfrak{H}^{2}$; its domain $\mathfrak{D}(T)$ is the set of all $f \in \mathfrak{G}$ such that $\{f, g\} \in T$ for some $g \in \mathfrak{H}$, and its range $\mathfrak{R}(T)$ is the set of all $g \in \mathfrak{H}$ such that $\{f, g\} \in T$ for some $f \in \mathfrak{H}$. For $f \in \mathfrak{D}(T)$ we put $T(f)=\{g \in \mathfrak{H} \mid\{f, g\} \in T\}$. A subspace $T$ in $\mathfrak{H}^{2}$ is the graph of a linear function if $T(0)=\{0\}$; in this case we say $T$ is an operator in $\mathfrak{H}$, and then we denote $T(f)$ by $T f$.

The adjoint $T^{*}$ of a subspace $T$ in $\mathfrak{H}^{2}$ is defined by

$$
T^{*}=\left\{\{h, k\} \in \mathfrak{H}^{2} \mid(g, h)=(f, k) \text { for all }\{f, g\} \in T\right\} .
$$

If $J$ is the unitary operator in $\mathfrak{S}^{2}$ given by $J\{f, g\}=\{g,-f\}$, then $T^{*}=\mathfrak{S}^{2} \ominus J T$, the orthogonal complement of $J T$ in $\mathfrak{S}^{2}$. This shows that $T^{*}$ is also a subspace in $\mathfrak{H}^{2}$.

If $T$ is a subspace in $\mathfrak{H}^{2}$, let $T_{\infty}=\{\{f, g\} \in T \mid f=0\}$. Then $T_{s}=T \ominus T_{\infty}$ is a closed operator in $\mathfrak{H}$, and we have the orthogonal decomposition $T=T_{s} \oplus T_{\infty}$, with $\mathfrak{D}\left(T_{s}\right)$ dense in $\mathfrak{H} \ominus T^{*}(0), \mathfrak{R}\left(T_{s}\right) \subset \mathfrak{H} \ominus T(0)$.

A symmetric subspace $S$ in $\mathfrak{H}^{2}$ is one satisfying $S \subset S^{*}$, and a selfadjoint subspace $H$ is a symmetric one such that $H=H^{*}$. If $H=H_{s} \oplus H_{\infty}$ is a selfadjoint subspace in $\mathfrak{S}^{2}$ we have the result (due to Arens, [1, Theorem 5.3]) that $H_{s}$, considered as an operator in $\mathfrak{H} \ominus H(0)$, is a densely defined selfadjoint operator there. This permits a spectral analysis of a selfadjoint subspace $H$, once its operator part $H_{s}$ and its purely multi-valued part $H_{\infty}$ have been identified.

If $S, S_{1}$ are symmetric subspaces in $\mathfrak{S}^{2}$ such that $S \subset S_{1}$, then $S_{1}$ is said to be a symmetric extension of $S$. In [3] (see also [2]) we described all symmetric and selfadjoint extensions of a symmetric subspace $S$ in $\mathfrak{S}^{2}$. In this note we characterize precisely, in terms of "generalized boundary conditions", those selfadjoint subspace extensions of a nondensely defined symmetric operator $S$ in $\mathfrak{H}$. Applications to ordinary differential operators will be indicated in a subsequent note. Detailed

AMS (MOS) subject classifications (1970). Primary 47B99, 47B25, 47A20.

Key words and phrases. Symmetric subspace, selfadjoint subspace, selfadjoint extension, symmetric operator, selfadjoint operator.

${ }^{1}$ This research was supported in part by NSF Grant No. GP-33696X. 
proofs will appear elsewhere.

We require from [3, Theorems 12 and 15] two characterizations of the selfadjoint extensions $H$ of a symmetric subspace $S$ in $\mathfrak{H}^{2}$. All such satisfy $S \subset H \subset S^{*}$; let $M=S^{*} \ominus S$.

TheOREM A. A subspace $H$ is a selfadjoint extension of $S$ in $\mathfrak{H}^{2}$ if and only if $H=S \oplus M_{1}$, where $M_{1}$ is a subspace of $M$ satisfying $J M_{1}=M \ominus M_{1}$.

Alternatively, such $H$ may be described in terms of the subspaces $M^{ \pm}=\left\{\{h, k\} \in S^{*} \mid k= \pm i h\right\}$. We have $M=M^{+} \oplus M^{-}$and the following result.

THEOREM B. $A$ subspace $H$ is a selfadjoint extension of $S$ in $\mathfrak{H}^{2}$ if and only if there exists an isometry $V$ of $M^{+}$onto $M^{-}$such that $H=S \oplus$ $(I-V) M^{+}$, where $I$ is the identity operator. Thus $S$ has a selfadjoint extension in $\mathfrak{H}^{2}$ if and only if $\operatorname{dim} M^{+}=\operatorname{dim} M^{-}$.

2. Selfadjoint extensions of nondensely defined symmetric operators. Let $S_{0}$ be a symmetric densely defined operator in $\mathfrak{H}$, and let $\mathfrak{S}_{0}$ be a subspace of $\mathfrak{G}$. Throughout this section we assume that

$$
\operatorname{dim} \mathfrak{H}_{0}=p<\infty, \quad \operatorname{dim} M_{0}<\infty, \quad M_{0}=S_{0}^{*} \ominus S_{0} .
$$

We define $S$ to be the operator in $\mathfrak{H}$ given by

$$
\mathfrak{D}(S)=\mathfrak{D}\left(S_{0}\right) \cap\left(\mathfrak{H} \ominus \mathfrak{H}_{0}\right), \quad S \subset S_{0} .
$$

This operator is not densely defined, and so its adjoint will be a subspace which is not an operator.

THEOREM 1. Let $S$ be defined by (2.2), where (2.1) is assumed. Then $S$ is a symmetric operator with $\mathfrak{D}(S)$ dense in $\mathfrak{H} \ominus \mathfrak{H}_{0}$, and

$$
\begin{gathered}
S^{*}=\left\{\left\{h, S_{0}^{*} h+\varphi\right\} \mid h \in \mathfrak{D}\left(S_{0}^{*}\right), \varphi \in \mathfrak{G}_{0}\right\}, \\
\operatorname{dim} M^{ \pm}=\operatorname{dim}\left(M_{0}\right)^{ \pm}+\operatorname{dim} \mathfrak{G}_{0} .
\end{gathered}
$$

Thus $S^{*}(0)=\mathfrak{H}_{0}$ and $S^{*}$ is the algebraic sum of $S_{0}^{*}$ and $\left(S^{*}\right)_{\infty}$. From (2.4) and Theorem B it follows that $S$ has selfadjoint extensions in $\mathfrak{H}^{2}$ if and only if $\operatorname{dim}\left(M_{0}\right)^{+}=\operatorname{dim}\left(M_{0}\right)^{-}$, that is, if and only if $S_{0}$ has selfadjoint extensions in $\mathfrak{h}$. We now assume $\operatorname{dim}\left(M_{0}\right)^{+}=\operatorname{dim}\left(M_{0}\right)^{-}=\omega$, and indicate how one can characterize any selfadjoint extension $H$ of $S$ in $\mathfrak{S}^{2}$ by means of "generalized boundary conditions". Theorem A implies that any such $H=S \oplus M_{1}$ can be thought of as $H=S^{*} \ominus J M_{1}$, where $\operatorname{dim} M_{1}=p+\omega$. Thus

$$
H=\left\{\{h, k\} \in S^{*} \mid(k, \alpha)-(h, \beta)=0 \text { for all }\{\alpha, \beta\} \in M_{1}\right\},
$$

and (2.3) implies that $H$ is the set of all $\left\{h, S_{0}^{*} h+\varphi\right\} \in S^{*}$ satisfying 


$$
\langle h \alpha\rangle-\left(h, \varphi^{\prime}\right)+(\varphi, \alpha)=0
$$

for all $\left\{\alpha, S_{0}^{*} \alpha+\varphi^{\prime}\right\} \in M_{1}$. Here we have introduced the abbreviation $\langle h \alpha\rangle=\left(S_{0}^{*} h, \alpha\right)-\left(h, S_{0}^{*} \alpha\right), h, \alpha \in \mathfrak{D}\left(S_{0}^{*}\right)$. By thinking of $H$ as in (2.5) we obtain the following precise characterization.

THEOREM 2. Let $H$ be a selfadjoint subspace extension of $S$ in $\mathfrak{H}^{2}$, with $\operatorname{dim} H(0)=s$. Let an orthonormal basis for $H(0)$ be $\varphi_{1}, \ldots, \varphi_{s}$, and suppose $\varphi_{1}, \ldots, \varphi_{s}, \varphi_{s+1}, \ldots, \varphi_{p}$ is an orthonormal basis for $S^{*}(0)=\mathfrak{H}_{0}$. Then $H$ is the set of all $\left\{h, S_{0}^{*} h+\varphi\right\} \in S^{*}$ such that

(i) $\left(h, \varphi_{j}\right)=0, j=1, \ldots, s$,

(ii) $\left\langle h \delta_{j}\right\rangle-\left(h, \zeta_{j}\right)=0, j=p+1, \ldots, p+\omega$,

(iii) $\varphi=c_{1} \varphi_{1}+\cdots+c_{s} \varphi_{s}+\sum_{k=s+1}^{p}\left[\left(h, \psi_{k}\right)-\left\langle h \gamma_{k}\right\rangle\right] \varphi_{k}, c_{j} \in C$ arbitwhere rary,

(a) $\gamma_{s+1}, \ldots, \gamma_{p} \in \mathfrak{D}\left(S_{0}^{*}\right)$

(b) $\delta_{p+1}, \ldots, \delta_{p+\omega} \in \mathfrak{D}\left(S_{0}^{*}\right)$ are linearly independent $\bmod \mathfrak{D}\left(S_{0}\right)$, and $\left\langle\delta_{j} \delta_{k}\right\rangle=0, j, k=p+1, \ldots, p+\omega$,

(c) $\zeta_{j}=-\sum_{k=s+1}^{p}\left\langle\delta_{j} \gamma_{k}\right\rangle \varphi_{k}, j=p+1, \ldots, p+\omega$,

(d) $\psi_{j}=\sum_{k=s+1}^{p}\left[E_{k j}-\frac{1}{2}\left\langle\gamma_{j} \gamma_{k}\right\rangle\right] \varphi_{k}, j=s+1, \ldots, p, E_{j k} \in C, E=\left(E_{j k}\right)$ $=E^{*}$.

Conversely, let $\varphi_{1}, \ldots, \varphi_{p}$ be an orthonormal basis for $\mathfrak{H}_{0}$, suppose $\gamma_{j}, \delta_{j}$ exist satisfying (a), (b), and $\zeta_{j}, \psi_{j}$ are defined by (c), (d). Then $H$ defined via (i)-(iii) is a selfadjoint extension of $S$ with $\operatorname{dim} H(0)=s$.

The operator part $H_{s}$ of $H$ is given by

$$
H_{s} h=Q_{0} S_{0}^{*} h+\sum_{k=s+1}^{p}\left[\left(h, \psi_{k}\right)-\left\langle h \gamma_{k}\right\rangle\right] \varphi_{k},
$$

where $Q_{0}$ is the orthogonal projection of $\mathfrak{H}$ onto $\mathfrak{H} \ominus H(0)$.

With appropriate interpretations, Theorem 2 remains valid in the three cases: $s=0, s=p$, and $\omega=0$. If $s=0$ then $H$ is an operator extension of $S$, and those operator extensions $H$ satisfying $S_{0} \subset H \subset S_{0}^{*}$ are obtained by taking $\gamma_{j}=0, E_{k j}=0$, which results in $\zeta_{j}=0, \psi_{j}=0$. Then

$$
\begin{aligned}
\mathfrak{D}(H) & =\left\{h \in \mathfrak{D}\left(S_{0}^{*}\right) \mid\left\langle h \delta_{j}\right\rangle=0, j=p+1, \ldots, p+\omega\right\}, \\
\left\langle\delta_{j} \delta_{k}\right\rangle & =0, \quad j, k=p+1, \ldots, p+\omega,
\end{aligned}
$$

which is the known characterization of such $H$. If $\omega=0$ and $s=p$, $H(0)=\mathfrak{H}_{0}$ and $H_{s} h=Q_{0} S_{0} h$. Thus, given any selfadjoint operator $S_{0}$ in $\mathfrak{H}$, with $\mathfrak{D}\left(S_{0}\right)$ dense in $\mathfrak{H}$, and subspace $\mathfrak{H}_{0} \subset \mathfrak{H}$, $\operatorname{dim} \mathfrak{H}_{0}<\infty$, the operator $H_{s}$ on $\mathfrak{H} \ominus \mathfrak{H}_{0}$ defined by $H_{s} h=Q_{0} S_{0} h$ is a densely defined selfadjoint operator. This is a result due to $\mathrm{W}$. Stenger [4, Lemma 1].

\section{REFERENCES}

1. R. Arens, Operational calculus of linear relations, Pacific J. Math. 11 (1961), 9-23. 
MR 23 \# A517.

2. E. A. Coddington, Multi-valued operators and boundary value problems, Lecture Notes in Math., No. 183, Springer-Verlag, Berlin, 1971.

3. ㄴ. Extension theory of formally normal and symmetric subspaces, Mem. Amer. Math. Soc. No. 134 (1973).

4. W. Stenger, On the projection of a selfadjoint operator, Bull. Amer. Math. Soc. 74 (1968), 369-372. MR 36 \# 3142.

Université de Paris Vi, Paris, France

Department of Mathematics, University of California, Los Angeles, California 90024 